Schweizerisches Jahrbuch für Entwicklungspolitik

16 | 1997

Umwelt und Entwicklung, Rio : Fünf Jahre danach

\title{
3. Innenpolitik/Ausenpolitik
}

\section{(2) OpenEdition \\ 1 Journals}

Electronic version

URL: http://journals.openedition.org/sjep/737

DOI: 10.4000/sjep.737

ISSN: 1663-9677

Publisher

Institut de hautes études internationales et du développement

\section{Printed version}

Date of publication: 1 mars 1997

Number of pages: $97-116$

ISSN: 1660-5926

\section{Electronic reference}

« 3. Innenpolitik/Ausenpolitik », Schweizerisches Jahrbuch für Entwicklungspolitik [Online], 16 | 1997

Online erschienen am: 28 August 2012, abgerufen am 08 September 2020. URL : http://

journals.openedition.org/sjep/737 ; DOI : https://doi.org/10.4000/sjep.737

(c) The Graduate Institute 


\section{INNENPOLITIK/AUSSENPOLITIK}

\section{DIE SCHWEIZERISCHE ASYLPOLITIK UND MIGRATIONSFRAGEN}

Die rechtliche Basis der schweizerischen Asylpolitik bildet das Asylgesetz aus dem Jahre 1979, das - wie kaum ein anderes Gesetz - bereits vier Teilrevisionen unterzogen wurde, um den Veränderungen im Asylbereich Rechnung zu tragen. Zur Zeit ist eine Totalrevision des Asylgesetzes im Gange. Grundtenor des Revisionsprozesses ist die restriktivere Ausgestaltung der Asylpraxis. Kernpunkt der Revision ist die Schaffung der Gesetzesgrundlage für die Gewährung eines vorübergehenden Schutzes für sog. Gewaltflüchtlinge. Die gestellten Asylgesuche in der Schweiz schwankten in den letzten zehn Jahren zwischen 8000 und 42'000 Asylanträgen pro Jahr. Im Jahre 1995 waren es 17'021 Gesuche; bis Ende August 1996 stellten insgesamt 11'069 Personen ein Asylgesuch. Am 1. Dezember 1996 kam die Volksinitiative "Gegen die illegale Einwanderung “ zur Abstimmung. Sie wurde mit 53,6\% Nein-Stimmenanteil verworfen. Der Bund erarbeitet zur Zeit eine Migrationspolitik mit dem Ziel, ein eigentliches Migrationsgesetz vorzulegen, welches den Fluchtursachen und den Fluchtbedingungen einerseits sowie der wirtschaftlichen Lage auf dem Schweizer Arbeitsmarkt und der finanziellen Situation des Bundes andererseits Rechnung tragen soll. Der Grundtenor hier ist eine migrationshemmende Politik und eine bessere Vernetzung der durch Einwanderung betroffenen Politikbereiche.

Der grösste Teil der Flüchtlinge - weltweit auf 50 Millionen Menschen geschätzt - flieht vor Krieg und kriegerischen Auseinandersetzungen und vor Situationen wirtschaftlicher Not. Die grössten Flüchtlingsstöme bewegen sich in den Entwicklungsländern, nur ein kleiner Teil dieser Migration gelangt bis in die Industrieländer. Der weitaus grösste Teil der Flüchtlinge bleibt im Süden und verschärft die Entwicklungsprobleme der betroffenen Region, wie dies der Bundesrat verschiedentlich im Kontext der Entwicklungszusammenarbeit und der humanitären Hilfe hervorhebt.' Betreffend die Schweiz kommt der weitaus grösste Teil der Flüchtlinge aus den Konflikt- und Kriegsgebieten Ex-Jugoslawien, Sri Lanka und der Türkei.

\section{$\square$ Asylgesuche}

Die Tabelle über die Asylgesuche zeigt, dass sich die 1995 eingereichten 17'021 Asylgesuche im Rahmen der Jahre mit tieferen Asylzahlen bewegen. Ausnahmen bildeten die Jahre 1990 und 1991, als Gesuche aus dem Kriegsgebiet im ehemaligen Jugoslawien die Zahl der Asylsuchenden stark erhöhte.

\footnotetext{
Siehe beispielsweise das Kapitel Die Migrationsbewegungen in der Botschaft über die technische Zusammenarbeit und der Finanzhilfe zugunsten von Entwicklungsländern vom 20.4.1994. Im Jahrbuch 1996 wurden die Aspekte Migrationspolitik und Entwicklungszusammenarbeit sowie der Migrationsbericht von Peter Arbenz ausführlich besprochen (S. 96ff.).
} 
Tabelle Nr. 15

\begin{tabular}{|c|c|c|c|c|c|c|c|c|c|c|}
\hline \multicolumn{11}{|c|}{ In der Schweiz eingereichte Asylgesuche $1985-1995$} \\
\hline 1985 & 1986 & 1987 & 1988 & 1989 & 1990 & 1991 & 1992 & 1993 & 1994 & 1995 \\
\hline 9703 & 8546 & 10 '913 & $16^{\prime} 726$ & $24^{\prime} 425$ & $35^{\prime} 836$ & $41^{\prime} 692$ & $17 ’ 960$ & $24 ’ 739$ & $16^{\prime} 134$ & $17^{\prime} 021$ \\
\hline
\end{tabular}

\begin{tabular}{lllr}
\hline Herkunftsland Gesuche 1995 & & \\
\hline Bundesrepublik Jugoslawien & 5491 & Sri Lanka & 1024 \\
\hline Bosnien & 3534 & Angola & 493 \\
\hline Türkei & 1293 & Somalia & 478 \\
\hline
\end{tabular}

Quelle : EJDP/Bundesamt für Flüchtlinge.

Die meisten Aslygesuche werden von Personen gestellt, welche aus Regionen mit kriegerischen Konflikten fliehen: Ex-Jugoslawien, die Türkei (Kurdenkonflikt), Sri Lanka sowie afrikanische Länder mit politischen Unruhen (Angola, Somalia, Zaire, Algerien). 1995 waren die wichtigsten Herkunftsländer:

Die durchschnittliche Anerkennungsquote lag bei 14,9\% 1995 und bei 12,5\% 1994.

Im Rahmen des Asylverfahrens hielten sich Ende 1995 125'448 Personen in der Schweiz auf, 24'581 davon waren anerkannte Flüchtlinge. Nur rund 35 Prozent aller Asylsuchenden und vorläufig Aufgenommenen haben eine Arbeitsstelle. Die Fürsorgeaufwendungen betrugen 1995 insgesamt 812 Millionen Franken; in diesem Betrag sind die Fürsorgeaufwendungen für die Asylbewerber und die Flüchtlinge, die Verwaltungskosten der Kantone, die übrigen Ausgaben des Bundesamtes für Flüchtlinge enthalten.

\section{$\square$ Rückweisung/Rückkehrhilfe}

Seit 1984 sind rund 30'000 asylsuchende Personen aus Sri Lanka in die Schweiz gekommen. Der grösste Zustrom erfolgte in den Jahren 1989 bis 1991 . Wegen des Bürgerkrieges im Norden und Osten des Inselstaates wurden bis 1993 keine Wegweisungen vollzogen, mit Ausnahme der Rückführungen von Personen, die sich "deliktisch, rechtsmissbräuchlich oder dissozial"“2 verhalten haben. Mit Sri Lanka hat die Schweiz im Januar 1994 ein auf zwei Jahre befristetes Rückkehrabkommen unterzeichnet, das abgewiesenen Asylbewerbern eine Rückkehr in die Heimat " in Sicherheit und Würde" ermöglichen soll. Das Abkommen soll für zwei weitere Jahre verlängert werden. Allerdings haben weniger abgewiesene Tamilen die Rückkehrhilfe nach Sri Lanka beansprucht, als dies das Bundesamt für Flüchtlinge angenommen hatte; es kehrten 1994/95 insgesamt 385 Personen im Rahmen dieses Rückkehrabkommens nach Sri Lanka zurück; davon rund ein Drittel freiwillig ${ }^{3}$. Die Rückführungen mussten zeitweilig unterbrochen werden, weil die Situation in Sri Lanka eine Wegweisung gemäss den Prinzipien des

2 Pressemitteilung des Bundesamtes für Flüchtlinge vom 18.12.1995, Der Bundesrat verlängert die Vereinbarung mit Sri Lanka über die Rückführung abgewiesener Asylsuchender.

3 Diese Zahlen veröffentlichte das Bundesamt für Flüchtlinge in der Pressemitteilung vom 18.12.1995. 
Abkommens nicht zuliessen. Mit der Türkei wird ebenfalls eine Form von Rückkehrhilfe erprobt: 100 Personen erhalten ihre Reisekosten vergütet und einen Wiedereingliederungsbeitrag von 455 Franken pro Monat während sechs Monaten; sie erhalten zweimal Besuch einer Vertretung der Internationalen Organisation für Migration. In den Kantonen sollen Rückkehrberatungsstellen errichtet werden, nachdem mit einer solchen Stelle in Genf gute Erfahrungen gemacht worden sind.

Das UNHCR kritisierte die Rückkehr-Planung der Schweiz für Flüchtlinge aus Angola und aus Bosnien. Die Rückweisung (mit Rückkehrhilfe) nach Bosnien hat die Schweiz nach Ansicht der UNHCR-Direktorin zu früh veranlasst. Der Bundesrat hat im April 1996 beschlossen, dass rund 21'000 bosnische Flüchtlinge bis April bzw. August 1997 nach Bosnien zurückkehren sollen. Er hat gleichzeitig betont, dass im Verlaufe des Monats März 1997 eine Überprüfung der Lage vor Ort vorgenommen wird, um zu entscheiden, ob an diesen Terminen festgehalten werden wird oder nicht. Seit Mitte August 1996 ist eine operationelle Rückkehrhilfe nach Bosnien im Gange; diese hat bis Ende 1996 über 2000 Bosnierinnen und Bosnier zur Rückkehr bewogen.

In Angola sei die politische Situation und Sicherheitslage nicht gefestigt, und das UNHCR empfahl, die Rückschaffung hinauszuschieben. Der Bundesrat hielt in beiden Fällen an seiner Ausschaffungspraxis fest.

Im Falle abgewiesener Gesuche von Kosovo-Albanern hat der Bundesrat die Ausreisefrist bis Ende März 1997 verlängert, weil sich die Führung in Belgrad weigert, die Rückkehrer aufzunehmen. Zusammen mit anderen europäischen Staaten drängt die Schweiz Rest-Jugoslawien zum Abschluss eines Rückübernahmeabkommens.

\section{$\square$ Initiative gegen die illegale Einwanderung}

Ziel dieser Initiative der Schweizerischen Volkspartei (SVP) ist die massive Reduktion der Asylgesuche durch das Mittel des Nicht-Eintretens auf die Gesuche aller illegal eingereisten Personen. Durchschnittlich betrifft dies $85 \%$ aller in der Schweiz um Asyl nachfragenden Personen. Die Initiative ist unter dem Eindruck der hohen Asylgesuche anfangs der neunziger Jahre lanciert worden und in der Zwischenzeit durch die Entwicklung überholt, weshalb der Bundesrat die Initiative zur Ablehnung empfahl. Der Bundesrat setzt auf die Harmonisierung der Asylpolitik auf internationalem Niveau, vor allem mit den Ländern der Europäischen Union. Er argumentierte, dass die Initiative die schweizerische Asylpolitik zu stark festbinden wolle und der Ausgestaltung einer zukunftsgerichteten, international koordinierten Asylpraxis im Rahmen einer umfassenden Migrationspolitik keinen Raum mehr lasse. Der Nationalrat hatte die Initiative mit 139 zu 36 Stimmen und der Ständerat mit 35 zu 3 Stimmen abgelehnt. In der Volksabstimmung wurde sie mit 53,6\% Nein-Stimmen abgelehnt. Bei den Ständen fiel das Resultat noch knapper aus: 11 Kantone stimmten der Initiative zu, 12 Kantone lehnten sie $a b$.

\section{$\square$ Revision des Asylgesetzes}

Die wichtigsten Elemente des bundesrätlichen Entwurfs zur Totalrevision des Asylgesetzes sind die Schaffung von Bestimmungen über die vorübergehende Aufnahme von Kriegsflüchtlingen (Gewaltflüchtlinge), die Vereinfachung der 
Fürsorgestrukturen und eine Senkung der Fürsorgekosten sowie die Aufnahme von Datenschutzbestimmungen.

Gewaltflüchtlinge: Der Entwurf des Bundesrates für das revidierte Asylgesetz sieht vor, dass die Regierung einen kollektiven Entscheid betreffend Anzahl von Personen fällen kann, welche aus einem bestimmten Gebiet vor Kriegswirren in die Schweiz fliehen und hier vorübergehenden Schutz erhalten sollen, ohne dass sie das aufwendige individuelle Asylverfahren durchlaufen müssen (Kapitel 4 der Botschaft 95.088). In der Botschaft zur Totalrevision bezeichnet der Bundesrat das Kapitel 4 als Kernstück der Vorlage. Das Konzept der Gewaltflüchtlinge basiert gemäss Bundesrat auf folgenden Elementen:

1. Der Bundesrat trifft den Grundsatzentscheid, ob und wie viele Personen vorübergehender Schutz gewährt wird.

2. Bei der Aufnahme Schutzbedürftiger in der Schweiz steht nicht ihr dauernder Aufenthalt im Vordergrund, sondern die Rückkehr in ihren Heimat-oder Herkunftsstaat, sobald sich die Möglichkeit dafür bietet.

3. Das Verfahren wird so gestaltet, dass - im Gegensatz zur heutigen Lösung bei der gruppenweisen vorläufigen Aufnahme (...) - die Asylbehörden von der Durchführung eines aufwendigen Individualverfahrens entlastet werden (Botschaft 95.088, Übersicht, S.2).

Die BODS (Bewegung für eine offene, demokratische und solidarische Schweiz) und die Asylkoordination Schweiz sehen in dieser Vereinfachung der Asylverwaltung auch eine Gefahr: Wenn eine asylsuchende Person zu einer Gruppe gehöre, welche unter die kollektive Schutzbestimmung falle, werde ihr kein individuelles Asyl mehr gewährt, solange der kollektive Schutz gelte. Die Asylbewegung setzt sich dafür ein, dass Gewaltflüchtlingen weiterhin der Zugang zum individuellen Asylverfahren offensteht und dass sie Asyl bekommen sollen, wenn sie eine persönliche Verfolgung nachweisen können. In Artikel 66 der Vorlage sieht der Bundesrat den Zugang zum individuellen Asylverfahren "in Ausnahmefällen" vor.

Sichere Länder: 1990 wurde mit einem allgemeinen Bundesbeschluss das "Safe country"-Konzept eingeführt, wonach auf Asylgesuche aus verfolgungssicheren Staaten nicht eingetreten wird. Der Bundesrat entscheidet über die Aufnahme bzw. den Widerruf eines Landes auf diese Liste. Sie enthält folgende Länder (Stand September 1996): Bulgarien, Rumänien, Albanien, Ghana, Senegal, Gambia, Indien. Im Falle von Gambia und Ghana wird der Bundesrat im Frühjahr 1997 den Safe-Country-Status neu überprüfen. Die Praxis der Liste der sicheren Länder wurde mittlerweilen durch fast alle EU-Staaten übernommen.

\section{$\square$ Migrationspolitik}

1993 erteilte das Parlament dem Bundesrat den Auftrag, eine Migrationspolitik $\mathrm{zu}$ formulieren und Verfassungsbestimmungen sowie ein Migrationsgesetz auszuarbeiten. Ziel dieser Politik ist es, den Zuwachs der ausländischen Bevölkerung weiter zu reduzieren und die Bereiche Asyl-, Ausländer- und Arbeitsmarktpolitik besser aufeinander abzustimmen. Als Basis für die Diskussion über eine Migrationspolitik gab der Bundesrat den Bericht über eine schweizerische Migrationspolitik in Auftrag (Verfasser Peter Arbenz, ehemals Direktor des BFF). ${ }^{4}$ Der Bericht hat zahlreiche Reaktionen ausgelöst; 72 Vernehmlassungen 
sind eingereicht worden. In einem zentralen Punkt decken sich die Meinungen weitgehend: fehlende Kohärenz zwischen den einzelnen Politikbereichen, ungenügende Koordination zwischen den Ämtern und fehlender Einbezug der Öffentlichkeit. Weit auseinander gehen die Urteile in anderen wichtigen Bereichen. So wird beispielsweise das ausländerpolitische Drei-Kreise-Modell - seit 1995 im Rahmen des Ausländergesetzes angewandt - von der Eidgenössischen Kommission gegen Rassismus als rassismusfördernd bezeichnet. Die Vorstellungen über das weitere Vorgehen zur Formulierung einer Migrationspolitik gehen z.T. diametral auseinander, was die Erarbeitung eines nationalen Konsenses für die Gestaltung der neuen Migrationspolitik nicht zu einer leichten Aufgabe macht. Eine Expertenkommission ist mit der Formulierung von Vorschlägen für eine künftige Politik betraut worden; Mitte 1997 sollen diese vorliegen. Die Vorgaben des Bundesrates sind: eine bessere Vernetzung von Asyl-, Ausländer-, Arbeitsmarkt- und Entwicklungshilfepolitik.

\section{QUELLEN}

Botschaft zur Totalrevision des Asylgesetzes sowie zur Änderung des Bundesgesetzes über Aufenthalt und Niederlassung der Ausländer vom 4.12.1995 (95.088).

Bundesamt für Flüchtlinge, Asylstatistik 1995, Liste der verfolgungssicheren Staaten - Safe Countries, Asylon Aktuell (Ausgaben 1995 und 1996).

Volksabstimmung vom 1. Dezember 1996 - Erläuterungen des Bundesrates

EJPD/Bundesamt für Flüchtlinge, Pressemitteilung vom 18.12.1995.

Grüne Position zur Migrationspolitik, Hrsg. Grüne Partei der Schweiz, Bern, Oktober 1996.

BODS-Rundbrief Nr. 2/Juni 1996: Worum es beim neuen Asylgesetz geht.

BODS-Rundbrief Nr. 3/September 1996: Abstimmung Asylinitiative.

Asylgesetz: Total überholt, Stellungnahme zur Totalrevision des Asylgesetzes/Argumentenkatalog, Hrsg. Asylkoordination Schweiz, Bern.

Sri Lanka - Blinde Rückschaffungspolitik, in: Amnesty Magazin Nr. 2/Februar 1996.

"Enge Spielräume in der Migrationspolitik“" in: Neue Zürcher Zeitung, 15,5.1996.

"Expertenkommission für Migrationspolitik" in: Neue Zürcher Zeitung, 11.6.1996.

Kirche und Asyl. Legitimer Widerstand im Rechtsstaat? Hrsg. Muriel Beck Kadima und Jean-Claude Huot, Institut für Sozialethik des SEK, Zürich 1996.

\section{KAPITALFLUCHT, KORRUPTION UND GELDWÄSCHEREI}

Während der Herbst- und Wintermonate 1996/97 war die Schweizer Politik geprägt von der Affäre um die nachrichtenlosen Vermögen und das Nazi-Gold, die den Finanzplatz Schweiz ins Visier nahm und dessen Ansehen arg strapazierte, da er nach Ansicht gewisser Kreise als regelrechte Drehscheibe für Gelder aus dem organisierten Verbrechen fungierte. Allerdings wurden sowohl auf nationaler als auch auf internationaler Ebene eine Reihe von Massnahmen zur Bekämpfung der Kriminalität im grossen Stil ergriffen. Die Eidgenössischen Räte hiessen eine Revision des Gesetzes über die internationale Rechtshilfe gut, die allerdings von einigen Staatsanwälten und Richtern als zu zaghaft bezeichnet wurde. Der Bundesrat legte eine Botschaft über die Bekämpfung der Geldwäscherei vor. Darüber hinaus nimmt die Schweiz an der Tätigkeit der Aktionsgruppe der OECD gegen Geldwäscherei (FATF) teil. Transparency International, eine Nichtregierungsorganisation, die sich für die Bekämpfung der Korruption im internationalen Handel einsetzt, gründete eine Sektion in der Schweiz.

\footnotetext{
${ }^{4}$ Dieser Bericht ist im Jahrbuch 1996 ausführlich besprochen.
} 


\section{$\square$ Internationale Rechtshilfe}

Auf Anordnung des Bundesrates wird das revidierte Bundesgesetz über internationale Rechtshilfe in Strafsachen am 1. Februar 1997 in Kraft treten. ${ }^{1}$ Während der Herbstsession 1996 hat der Nationalrat das revidierte Rechtshilfegesetz endgültig verabschiedet. Die wichtigste Neuerung besteht darin, dass künftig im Rahmen eines Verfahrens nur noch eine einzige Beschwerdemöglichkeit besteht. Einzig das letztinstanzliche Urteil kann angefochten werden, die Möglichkeit vorbeugender Beschwerden besteht nicht mehr. Ausserdem werden die Schweizer Richter in Zukunft ihren ausländischen Kollegen Beweismittel und Informationen unter gewissen Bedingungen direkt übermitteln können. Diese Möglichkeit, den Verfahrensweg zu umgehen, besteht allerdings nicht im Zusammenhang mit Beweisen, die dem Bankgeheimnis unterliegen. Im Laufe der Parlamentsdebatte traten weitreichende Divergenzen zwischen den beiden Kammern zutage. Während der Wintersession 1995 hatte der Nationalrat entgegen der Meinung des Bundesrates entschieden, eine Frist von neun Monaten für die Behandlung eines Rechtshilfegesuchs zu setzen. Der Ständerat schloss sich diesem Entscheid nicht an.

Diese Gesetzesrevision stiess in weiten Kreisen auf wenig Begeisterung. Einerseits erklärten sich die Untersuchungsrichter, die sich mit der Rechtshilfe befassen, enttäuscht über die vom Parlament verabschiedete Revision. In den am stärksten betroffenen Kantonen (Zürich, Genf und Tessin), wo die Banken in ausländische Finanz- und Politskandale verwickelt worden waren, erklärten einige Justizbeamte, sie erwarteten keine grundlegenden Änderungen in der Praxis. Die Aufrechterhaltung einer kantonalen Beschwerdeinstanz, deren Urteil möglicherweise vom Bundesgericht angegriffen werde, sei nach wie vor der Hauptgrund, weshalb Rechtshilfeersuchen aus dem Ausland nicht mit der gebotenen Eile bearbeitet werden können. Die Erklärung von Bern ${ }^{2}$ hingegen begrüsste die Fortschritte, die dank der Revision erzielt, wurden, insbesondere die Einschränkung der Beschwerdemöglichkeiten auf eine einzige Instanz. Sie bedauert jedoch, dass das Gesetz keine Frist für die Bearbeitung des Verfahrens vorsieht, und dass die Rechtshilfe nicht auf weitere Bereiche ausgeweitet wurde (Steuerflucht).

继 1996 Internationale Rechtshilfe, S. 109.

Ende Oktober 1996 unterzeichneten Bundesrat Arnold Koller und der französische Justizminister Jacques Toubon einen Zusatzvertrag zum Europäischen Übereinkommen über die Rechtshilfe in Strafsachen. Ziel dieses Zusatzvertrags ist die Vereinfachung und Beschleunigung der Bearbeitung von Rechtshilfeersuchen zwischen den beiden Staaten. Die Gerichtsbehörden werden in Zukunft ihre Rechtshilfeersuchen direkt an den Generalstaatsanwalt des örtlich zuständigen Appellationsgerichts richten können und müssen sich nicht mehr wie bisher zuerst an das französische Justizministerium wenden. Der direkte Austausch von Rechtshilfeersuchen zwischen schweizerischen und französischen Richtern wird auch weiterhin in dringlichen Fällen möglich sein (vgl. Art. $15 \S 2$ des Übereinkommens).

1 Bundesgesetz über internationale Rechtshilfe in Strafsachen, Änderung vom 4. Oktober 1996, in: Bundesblatt, Nr. 41, Band IV, 15. Oktober 1996.

2 Die Erklärung von Bern hatte 1991 ein von über 200 NRO mitgetragenes Manifest mit dem Titel "Für eine Schweiz ohne Fluchtgelder" verfasst, in welchem sie eine Begrenzung des Verfahrens auf sechs Monate, eine Ausweitung des Anwendungsbereiches der Rechtshilfe in Strafsachen und ein auf Bundesebene zentralisiertes Rechshilfeverfahren für Fälle von Kapitalflucht im Zusammenhang mit ehemaligen Staatschefs forderte. 
Darüber hinaus hat die Schweiz im Juli 1996 je ein Rechtshilfeabkommen mit Peru und Ecuador paraphiert. Beide Abkommen sehen die direkte Übermittlung von Rechtshilfeersuchen zwischen den Zentralbehörden der Schweiz und des jeweiligen Staates vor. Erwähnenswert ist, dass es sich dabei um die ersten Abkommen dieser Art zwischen der Schweiz und südamerikanischen Staaten handelt.

\section{$\square$ Botschaft über die Bekämpfung der Geldwäscherei}

Das organisierte Verbrechen stellt heute eine der grossen Gefahren für Wirtschaft und Gesellschaft dar. Die kriminellen Organisationen verfügen aus ihrer verbrecherischen Tätigkeit (Drogen- und Waffenhandel, Prostitution von Frauen und Kindern etc.) über riesige Geldsummen, die für sie jedoch weitgehend nutzlos sind, solange ihre verbrecherische Herkunft nachgewiesen werden kann. Erst wenn diese Gelder ,gewaschen“ sind, können sie gefahrlos in den Wirtschaftskreislauf einfliessen. Ab diesem Zeitpunkt potenzieren sie die wirtschaftliche Macht des organisierten Verbrechens. Die gewaschenen Gelder können unverdächtig investiert werden und verhelfen den Verbrecherorganisationen so zu einem Mantel der Unbescholtenheit. Es besteht die konkrete Gefahr, dass wesentliche Teile ganzer Volkswirtschaften unter die Kontrolle des organisierten Verbrechens geraten.

Seit mehreren Jahren arbeiten Parlament und Bundesrat an einer Verschärfung der gesetzlichen Regelungen, die es erlauben soll, diese neuen Formen der Kriminalität effizienter zu bekämpfen. In weniger als zehn Jahren wurde das Instrumentarium erheblich erweitert. Am 1. August 1990 sind Art. $305^{\text {bis }}$ und $305^{\text {ter }}$ des Schweizerischen Strafgesetzbuches (StGB) bezüglich geldwäschereirelevanter Machenschaften und unsorgfältigen Finanzgebarens in Kraft getreten. Die strafrechtlichen Tatbestände sind am 1. August 1994 ergänzt worden: Ein zweiter Massnahmenkatalog zur Bekämpfung des organisierten Verbrechens erlaubt es, die von einer kriminellen Organisation kontrollierten Vermögenswerte zu konfiszieren (Art. 59 Abs. 3 StGB) und die Beteiligung an einer kriminellen Organisation sowie deren Unterstützung ausdrücklich zu bestrafen (Art. 260 ${ }^{\text {ter }} \mathrm{StGB}$ ), und legt im Strafgesetz das Melderecht des Finanzintermediärs im Zusammenhang mit verdächtigen Finanztransaktionen fest (Art. 305 ${ }^{\text {ter }}$, Abs. 2 StGB).

Im Juni 1996 legte der Bundesrat ein weiteres Gesetzespaket vor, welches die 1990 bzw. 1994 in Kraft getretenen Tatbestände ergänzen soll. In seiner Botschaft ${ }^{3}$ schreibt der Bundesrat: "Das Geldwäschereigesetz (GwG) auferlegt den im Finanzsektor tätigen natürlichen und juristischen Personen Sorgfaltspflichten sowie organisatorische Massnahmen zur Verhinderung der Geldwäscherei. Zudem werden die Finanzintermediäre verpflichtet, den Strafverfolgungsbehörden Meldung zu erstatten und verdächtige Vermögenswerte zu sperren, sobald ein begründeter Verdacht auf Geldwäscherei vorliegt."

Dieser Gesetzesentwurf füllt eine Lücke aus. Im Gegensatz zu den Banken, Börsen, Effektenhändlern, Versicherungen und Anlagefonds fehlt eine Aufsicht über die übrigen Finanzintermediäre durch den Bund. Gemäss dem Gesetzesentwurf unterstehen auch alle Tätigkeiten des Nichtbankenbereichs der Sorgfaltspflicht,

${ }^{3}$ Botschaft zum Bundesgesetz zur Bekämpfung der Geldwäscherei im Finanzsektor (Geldwäschereigesetz GwG), in: Bundesblatt 1996, Bd. 3, S. $1101 \mathrm{ff}$. 
die darin besteht, die Identität des Kunden und der wirtschaftlich Berechtigten der betreffenden Vermögenswerte festzustellen und die Finanztransaktionen zu dokumentieren (paper trail). Die Frage, welche Pflichten einem Finanzintermediär, der einen Fall von Geldwäscherei vermutet, auferlegt werden sollen, war umstritten. Weite Kreise befürworteten die ledigliche Sperrung der fraglichen Vermögenswerte. Der Bundesrat hat sich dennoch für die Meldepflicht entschieden. Dabei muss der Finanzintermediär seinen Verdacht einer dafür vorgesehenen Meldestelle innerhalb des Bundesamtes für Polizeiwesen mitteilen, welche daraufhin bei Bedarf die Strafverfolgungsbehörden einschaltet und die unvermittelte Sperrung der fraglichen Vermögenswerte veranlasst.

In seiner Botschaft weist der Bundesrat ausserdem darauf hin, dass gemäss internationaler Empfehlungen (Financial Action Task Force on Money Laundering der OECD, FATF) die Meldepflicht Teil des internationalen Instrumentariums zur Bekämpfung der Geldwäscherei ist. Im März 1993 war in der Schweiz eine Überprüfung der Umsetzung der Empfehlungen der FATF durchgeführt worden. Dabei hatte sich herausgestellt, dass die Schweiz bei der Bekämpfung der Geldwäscherei im internationalen Vergleich gut dasteht. Im Zusammenhang mit Finanzinstituten des Nichtbankenbereichs waren allerdings einige Lücken zutage getreten. Im Bericht der FATF war der Vorentwurf des Geldwäschereigesetzes erwähnt und auf die Notwendigkeit einer möglichst raschen Inkraftsetzung des Gesetzes hingewiesen worden. Die Meldepflicht, die seitens der schweizerischen Finanzkreise heftigsten Widerstand hervorrief, ist in der Gesetzgebung aller westeuropäischen Staaten und derjenigen der USA enthalten. Im November 1996 trat die Kommission für Rechtsfragen des Nationalrates zusammen und verabschiedete einstimmig einen Text, der eine Verschärfung des Gesetzesentwurfs der Regierung fordert. Die Kommission schlägt vor, die Verletzung der Meldepflicht gegenüber den Behörden mit einer Busse von maximal Fr. 200’000.- zu bestrafen. Es wird sich zeigen, ob das Parlament dem Vorschlag der Kommission folgen wird: Die Botschaft des Bundesrates steht auf der Tagesordnung der Frühjahrssession 1997 des Nationalrates.

\section{$\square$ Bekämpfung der Korruption}

\section{Schlussbericht "Sicherheitsprüfungen und Korruption"}

In den vergangenen Jahren sind in den Industrieländern (Italien, Frankreich, Spanien) zahlreiche Korruptionsfälle im Zusammenhang mit Politikern und hohen Beamten bekanntgeworden. Die Schweiz stellt dabei keine Ausnahme dar. Einige Fälle, beispielsweise jene um den ehemaligen Vorsteher des Patentamtes des Kantons Zürich, Raphael Huber, oder um die Schweizerische Käseunion, erregten die Aufmerksamkeit der Medien und der Justiz. Diese Beispiele sind der Beweis dafür, dass die Korruption nicht nur in Entwicklungsländern vorkommt, sondern dass es sich dabei um ein Phänomen handelt, das die gesamte Gesellschaft und insbesondere die Bereiche Verwaltung, Wirtschaft und Politik betrifft.

Im Juli 1995 berief der Vorsteher des Eidgenössischen Justiz- und Polizeidepartementes, Bundesrat Arnold Koller, eine Arbeitsgruppe mit dem Namen "Sicherheitsprüfungen und Korruption" ein. Unter der Leitung von Urs von Däniken verfassten die Experten - vier Vertreter der Bundesverwaltung, zwei Vertreter der Kantonsbehörden und ein ständiger Experte der Zürcher Bezirksanwaltschaft - 
einen im November 1996 veröffentlichten Bericht ${ }^{4}$ mit Anträgen an die Adresse des Bundesrates. Die Arbeitsgruppe "beurteilt die Lage in der Schweiz nicht als alarmierend, hält aber mit Blick auf die vorhandenen negativen Signale und Rahmenbedingungen sowie die bekannten Entwicklungen im Ausland gezielte Massnahmen zur effizienten Bekämpfung der Korruption für erforderlich." Nach Ansicht der Experten sind die verfügbaren statistischen Daten - seit 1985 jährlich 40 bis 50 strafrechtliche Urteile - nicht repräsentativ. Es bestehen weiterhin Grauzonen, und in den wichtigsten Fällen ist entweder das Urteil noch hängig oder ein Berufungsverfahren im Gange. Es ist deshalb unmöglich, sich aufgrund der Zahlen ein Bild von der tatsächlichen Situation in der Schweiz zu machen. Die Arbeitsgruppe befürwortet deshalb konkrete Massnahmen aus dem repressiven, präventiven und administrativen Bereich, da es ihres Erachtens "entscheidend [ist], die Korruption an allen Fronten, d.h. in Verwaltung, Wirtschaft und Politik [...], gleichzeitig zu bekämpfen".

Die Empfehlungen zur Prävention und Bekämpfung der Korruption lassen sich wie folgt zusammenfassen:

a Ausarbeitung einer allgemeingültigen Musterregelung für die Annahme persönlicher Geschenke;

- Erstellung einer Liste der Funktionen, deren Inhaber massgeblich an den Entscheidungen über die Vergabe von Aufträgen oder im Beschaffungswesen beteiligt sind;

- Einführung des sogenannten Vieraugenprinzips und einer internen Kontrolle in den im öffentlichen Beschaffungswesen tätigen Bereichen der Verwaltung;

」 regelmässige Rotation der Verantwortlichen;

๑ Schaffung einer ständigen interdepartementalen Verwaltungskommission zur Lageverfolgung und -beurteilung.

Bei den Massnahmen zur Repression sind insbesondere zu erwähnen:

口 Pönalisierung der Geschenkgewährung an öffentliche Bedienstete;

- Erhöhung des im Schweizerischen Strafgesetzbuch festgelegten Strafmasses für Bestechung (bis zu fünf Jahren Zuchthaus) und Verlängerung der Verjährungsfristen;

- Aufnahme der aktiven Bestechung von ausländischen Beamten in den Katalog strafbarer Handlungen;

- Verbot des Steuerabzugs für Bestechungs- und Schmiergelder in Zusammenarbeit mit dem Ausland.

Während der Wintersession hiess der Ständerat die von Kurt Schuele (FDP, SH) eingereichte Motion ohne Gegenstimme gut, in welcher die Regierung aufgefordert wurde, das gesetzliche Instrumentarium der Bekämpfung dieses Phänomens anzupassen. In seiner Antwort auf die Motion versicherte Arnold Koller den Kantonsvertretern, dass bis Ende 1997 ein umfassendes Konzept über die Mittel zur Intensivierung der strafrechtlichen Korruptionsbekämpfung vorliegen werde. Dieses Konzept, das die Empfehlungen der Arbeitsgruppe "Sicherheitsprüfungen und Korruption" berücksichtigen soll, wird anschliessend in die Vernehmlassung geschickt.

${ }^{4}$ Schlussbericht der Arbeitsgruppe "Sicherheitsprüfungen und Korruption“, Eidgenössisches Justiz- und Polizeidepartement, Oktober 1996. Eine Zusammenfassung des Berichts liegt in französischer und deutscher Sprache vor. 
Steuerabzug für Bestechungsgelder

Die Praxis des Bundes, die sich auf ein Rundschreiben der eidgenössischen Steuerverwaltung aus dem Jahre 1946 beruft, wonach Bestechungsgelder für den Zuschlag von Aufträgen oder Bestellungen als erwerbsbedingte Auslagen vom Einkommen abgezogen werden können, wird von bestimmten Kreisen immer stärker unter Beschuss genommen. Diese Kreise sind der Ansicht, dass dieses Vorgehen einen sehr konkreten Anreiz zur aktiven Bestechung darstellt. Dies um so mehr, als Schmiergelder, wenn sie ans Ausland gezahlt werden, als dort üblichen Handelsbrauch toleriert werden. Die Wirtschaftskreise stehen einer grösseren Steuermoral nicht abgeneigt gegenüber, lassen aber durchblicken, dass diese Massnahmen "lebenswichtige Interessen der Exportindustrie berühren, indem sie einen Wettbewerbsnachteil schaffen". Zur Zeit ist die Bestechung ausländischer Beamter nur in den USA ausdrücklich strafbar, aber die Zahl der Länder, die den Steuerabzug für Bestechungsgelder abschaffen, nimmt ständig zu. Im April 1996 ermuntert die OECD ihre Mitgliedsländer, diese Politik ebenfalls einzuführen.

Ein von Werner Carobbio lancierter parlamentarischer Vorstoss, der die Abschaffung des Steuerabzugs für Bestechungsgelder forderte und der 1995 vom Nationalrat mit einer knappen Mehrheit angenommen worden war, löste in der Schweiz einen Besinnungsprozess aus. Nach langer Beratung schlug die Kommission für Wirtschaft und Abgaben des Nationalrats vor, den Steuerabzug für an Schweizer Beamte gezahlte Gelder abzuschaffen, ihn aber für Gelder, mit denen Private oder ausländische Beamte bestochen wurden, aufrechtzuerhalten.

$\square$ Weitere Initiativen im Kampf gegen die Korruption und das organisierte Verbrechen

a OECD: Bekämpfung der Korruption auf internationaler Ebene 1996 S. 112.

Die OECD hat sich für die Bekämpfung der Korruption auf internationaler Ebene, insbesondere im Bereich der Handelsbeziehungen, mehrere Ziele gesetzt. Ihr grösstes Anliegen ist die Förderung der einwandfreien Verwaltung im öffentlichen Sektor und in den Unternehmen, um unlauteren Wettbewerb beim Handel und bei den internationalen Investitionen zu unterbinden. Die OECD nahm ihre Tätigkeit zur Bekämpfung der Korruption 1989 auf Initiative der Vereinigten Staaten auf. 1994 verabschiedeten die Mitgliedsstaaten eine Empfehlung, in der sie sich verpflichten, konkrete und wirksame Massnahmen zur Bekämpfung der Korruption im Rahmen internationaler Handelsgeschäfte zu ergreifen. Im April 1996 wurde mit der Verabschiedung einer Empfehlung über den Abzug unerlaubter Zahlungen von der Steuer durch den OECD-Rat eine weitere Etappe abgeschlossen. Jene Länder, in denen soche Zahlungen von der Steuer abgezogen werden können, werden in der Empfehlung aufgefordert, ihre Gesetzgebung im Hinblick auf die Abschaffung dieser Möglichkeit zu revidieren. ${ }^{5}$

5 Communiqué de presse de l'OCDE, «Déclaration du Secrétaire général de l'OCDE à l'ouverture de la Conférence sur la corruption dans le cadre de transactions commerciales internationales», 6. November 1996. 
- Zwei Berichte des IWF über die Auswirkungen der Geldwäscherei

Zwei im Juli 1996 veröffentlichte Berichte des IWF weisen darauf hin, dass die Bewegungen von Geldern aus kriminellen Machenschaften die Volkswirtschaften jener Länder, in denen diese Mittel investiert werden, destabilisieren können. Ausserdem können diese aus der Sicht des Marktes ,unlogischen" Gelder die wirtschaftspolitischen Bemühungen der Regierungen zunichte machen. ${ }^{6}$

口 "Appel de Genève" von sieben europäischen Richtern unterzeichnet

Am 1. Oktober 1996 lancierten sieben hohe europäische Richter ${ }^{7}$ in Genf einen Appell zur Schaffung eines europäischen Gerichtsraums. Damit wollen sie die Hürden, die ihren Kampf gegen die Korruption behindern und ihren Handlungsspielraum beeinträchtigen, beseitigen. In ihrer Erklärung heisst es: "Um im Kampf gegen eine Kriminalität, die von den bestehenden unterschiedlichen Regelungen in den verschiedenen europäischen Ländern weitgehend profitiert, eine Chance zu erhalten, muss der überholte Protektionismus in polizeilicher und gerichtlicher Hinsicht überwunden werden. Die Schaffung eines europäischen Gerichtsraums, worin den Richtern bei der Recherche und beim Informationsaustausch im Zusammenhang mit laufenden Ermittlungen keine anderen Grenzen als diejenigen der Rechtsstaatlichkeit auferlegt werden, ist eine Notwendigkeit." Der Wortlaut des Appells ist in einem Buch des französischen Journalisten Denis Robert abgedruckt ${ }^{8}$. Das Buch enthält Interviews mit den sieben europäischen Richtern, in denen sie die Schwierigkeiten erläutern, denen sie in ihrer Arbeit begegnen.

- Schweizer Sektion von Transparency International

Transparency International ist eine im Mai 1993 gegründete NRO, die sich in der Bekämpfung der Korruption in internationalen Handelsgeschäften engagiert. Der Sitz der Organisation befindet sich in Berlin, und die nationalen Gruppen werden immer zahlreicher. Ziel der im November 1995 gegründeten Transparency Switzerland ${ }^{9}$ ist die Verhütung der Korruption, die Bekämpfung ihrer verschiedenen Formen und die Untersuchung ihrer Gründe und Auswirkungen. Sie will den Denkprozess und die Diskussion über diese Frage fördern, aber auch Lösungsvorschläge auf rechtlicher, politischer und wirtschaftlicher Ebene ausarbeiten.

\section{Aktivitäten der OECD-Arbeitsgruppe gegen Geldwäscherei (FATF)}

Die Schweiz nimmt an der Tätigkeit der Arbeitsgruppe der OECD gegen Geldwäscherei (FATF) aktiv teil. Die FATF wurde 1989 anlässlich des G7-Wirtschaftsgipfels in Paris ins Leben gerufen. Ihre Aufgabe ist die Prüfung von Massnahmen zur Bekämpfung der Geldwäscherei. Im April 1990 war ein Bericht mit 40 Empfehlungen zu diesem Thema veröffentlicht worden, welche 1993 erstmals

\footnotetext{
5 Communiqué de presse de l'OCDE, «Déclaration du Secrétaire général de l'OCDE à l'ouverture de la Conférence sur la corruption dans le cadre de transactions commerciales internationales», 6. November 1996.

${ }^{6}$ IMF Working Papers; von Tanzi, Vito: Money Laundering and the International System, Nr. 96/55; Quirk, Peter: Macroeconomic Implications of Money Laundering, $\mathrm{Nr}$. 96/66.

7 Berhard Bertossa (Schweiz), Edmundo Bruti-Liberati und Gherardo Colombo (Italien), Benoît Dejemeppe (Belgien), Baltazar Garzon Real und Carlos Jimenez Billarero (Spanien) sowie Renaud Van Ruymbeke (Frankreich).

8 La justice ou le chaos, Denis Robert, Stock, Paris 1996.

9 Transparency Switzerland, Haltbergstrasse 25, 8630 Rüti, Tel. 055/241 1117.
} 
revidiert wurden. Mitglieder der FATF sind 26 Staaten ${ }^{10}$ sowie zwei regionale Organisationen" ${ }^{11}$ die die wichtigsten Finanzzentren der Welt vertreten.

Die Aktivitäten der FATF während der siebten Arbeitsperiode (1995-96) konzentrierten sich im wesentlichen auf drei Achsen:

口 Überarbeitung der 40 Empfehlungen aus dem Jahre 1990. Die Mitglieder der FATF einigten sich auf mehrere grundlegende Änderungen. Erstens wurden die im Rahmen der Geldwäscherei verfolgten Straftaten über den Drogenhandel hinaus ausgeweitet. Zweitens wurde die Meldepflicht über verdächtige Kapitalbewegungen als obligatorisch erklärt und der Anwendungsbereich auf Unternehmen aus dem Nichtfinanzbereich erweitert. Die weiteren Änderungen beziehen sich unter anderem auf Scheinfirmen, die Präzisierung der Pflichten bei der Identifizierung der Kunden sowie auf die technologischen Neuerungen, die Überwachung des grenzüberschreitenden Bargeldverkehrs, die Technik der bewachten Botendienste und die Wechselstuben.

口 Selbstüberwachung der Durchsetzung der 40 Empfehlungen durch die Mitgliedsländer. Ein grosser Anteil der Tätigkeit der FATF entfällt auf die Sicherstellung der Durchsetzung der 40 Empfehlungen. Es wurde eine Reihe von Massnahmen zur Förderung der Selbstüberwachung erarbeitet, die die Mitgliedsstaaten bei dieser Aufgabe unterstützen sollen. Andererseits sind die Staaten aufgefordert, sich gegenseitig zu überwachen. Jedes Jahr werden einige Länder einer detaillierten Überprüfung unterzogen. Während der Arbeitsperiode 1995-96 waren Frankreich und Schweden an der Reihe. Zur Zeit startet die FATF eine zweite Überprüfungsserie, und die Schweiz wird 1997 erneut evaluiert werden.

口 Durchführung der Empfehlungen in den Nichtmitgliedsstaaten. Während der Periode 1995-96 hat die FATF eine Politik zur Evaluierung der Durchsetzung der Empfehlungen zur Bekämpfung der Geldwäscherei in den Nichtmitgliedsstaaten ausgearbeitet.

1996 S. 107.

\footnotetext{
QUELLEN

Bundesgesetz über internationale Rechtshilfe in Strafsachen, Änderung vom 4. Oktober 1996, in: Bundesblatt, Nr. 41, Band IV, 15. Oktober 1996.

Botschaft zum Bundesgesetz zur Bekämpfung der Geldwäscherei im Finanzsektor (Geldwäschereigesetz GwG), in: Bundesblatt 1996, Bd. 3, S. $1101 \mathrm{ff}$.

Schlussbericht der Arbeitsgruppe "Sicherheitsprüfungen und Korruption“, Eidgenössisches Justiz- und Polizeidepartement, Oktober 1996.

Rapport annuel 1995-1996, Groupe d'action financière sur le blanchiment de capitaux, 28. Juni 1996.

La justice ou le chaos, Denis Robert, Stock, Paris 1996.

Carla del Ponte, «La Suisse dans la sphère internationale de la criminalité», La vie économique, 2/1996

Vers un développement solidaire, Erklärung von Bern, Nr. 137, Januar 1997, Spezialdossier zur Korruption.

Finanzplatz-Informationen, 4/1996: "Geldwäscherei - vom Normalfall zur Ausnahme?"

Le Courrier, 2. Oktober 1996: «Genève accueille les juges en colère», 12. Dezember 1996: «Le Conseil des Etats active la lutte anticorruption".

Journal de Genève, 5. Oktober 1996: «Lutte contre le crime organisé, la Suisse se muscle, mais ...», 29. Oktober 1996: «Les juges suisses pourront parler des affaires aux procureurs français», 1. November 1996: «Taxer les pots-de-vin? Souhaitable, mais difficilement réalisable», 12. November 1996: «Le Conseil fédéral se donnera-t-il les moyens de combattre la corruption», 21. November 1996: «Loi anti-blanchissage les politiques balaient les objections des banquiers». Basler Zeitung, 15. April 1996: "Geldwäschereigesetz: Notwendigkeit eines Marschhaltes".
}

${ }^{10}$ Australien, Belgien, Dänemark, Deutschland, Finnland, Frankreich, Griechenland, Hongkong, Irland, Island, Italien, Japan, Kanada, Luxemburg, Neuseeland, Niederlande, Norwegen, Österreich, Portugal, Schweden, Schweiz, Singapur, Spanien, Türkei, Vereinigte Staaten, Vereinigtes Königreich.

${ }^{11}$ Europäische Kommission und Kooperationsrat der Golfstaaten. 
Neue Zürcher Zeitung, 7. August 1996: "Die Auswirkung der Geldwäscherei, Forderung nach schärferen Massnahmen in zwei IMF-Studien“.

Tages-Anzeiger, 18. März 1996: "Die Rechtshilfe soll beschleunigt werden“, 2. Oktober 1996: "Zaghafte Reform der Rechtshilfe".

Die Wochenzeitung, 6. September 1996, 'Wozdossier - Geldwäscherei“.

\section{WAFFENHANDEL}

Seit 1987 sind die Militärausgaben weltweit gesunken. Zwischen 1990 und 1995 hat das Verhältnis Militärausgaben/BIP in 90 Ländern abgenommen und in 25 Ländern zugenommen. Auf internationaler Ebene war das Jahr 1996 geprägt von zahlreichen Tagungen, die bezweckten, die Verwendung bestimmter konventioneller Waffen, insbesondere der Personenminen, einzuschränken oder zu verbieten. In der Schweiz haben der Nationalrat und der Ständerat am 13. Dezember 1996 der Revision des Bundesgesetzes über das Kriegsmaterial zugestimmt. Im Zentrum der Revision stand der Beschluss, die Pilatus-Flugzeuge aus dem Geltungsbereich des Gesetzes auszuschliessen, jedoch ihre Ausfuhr den Bestimmungen des Bundesgesetzes über die Kontrolle zivil und militärisch verwendbarer Güter zu unterstellen. Die gleiche Lösung wählte das Parlament für Maschinen und Werkzeuge, welche ausschliesslich für die Herstellung oder Wartung von Kriegsmaterial konzipiert sind. Damit schränkte das Parlament den Anwendungsbereich des Kriegsmaterialgesetzes im Vergleich zum Entwurf des Bundesrates ein. Die Lockerung entspricht den Wünschen der Wirtschaftskreise.

\section{$\square$ Waffenausfuhren der Schweiz}

Laut dem Bericht des SIPRI ist der Verkauf konventioneller Waffen von 1994 bis 1995 (22,8 Milliarden US\$) global um 0,2\% zurückgegangen. Die grössten Exporteure sind die Vereinigten Staaten, Russland, Deutschland, Grossbritannien und Frankreich. Die Schweiz stand 1995 nicht auf der Liste der 30 Hauptexporteure; ihre weltweiten Waffenexporte beliefen sich auf 141,17 Millionen Franken, was $0,15 \%$ der schweizerischen Gesamtexporte entspricht $(0,23 \%$ für das Jahr 1994). Nach Angaben des Eidgenössischen Militärdepartements handelt es sich um die niedrigste Zahl seit dem Inkrafttreten des Bundesgesetzes über das Kriegsmaterial im Jahr 1973. Zwischen 1994 und 1995 ist das Volumen der Exporte in Entwicklungsländer erheblich geschrumpft, wobei zu betonen ist, dass die Zahlen von einem Jahr zum anderen regelmässig starke Schwankungen aufweisen.

Der schweizerische Anteil an den Rüstungsausfuhren in Entwicklungsländer bewegte sich seit 1988 zwischen 0,11 und $0,29 \%$.

\section{$\square$ Revision des Bundesgesetzes über das Kriegsmaterial}

1996 setzen sich der Nationalrat und der Ständerat mit der Volksinitiative "für ein Verbot der Kriegsmaterialausfuhr" sowie mit dem Entwurf zu einem total revidierten Kriegsmaterialgesetz auseinander, welchen der Bundesrat in seiner Botschaft vom 15. Februar 1995 vorgelegt hatte (siehe Jahrbuch Schweiz - Dritte Welt 1996). 
Das Kriegsmaterialgesetz bezweckt, durch die Kontrolle der Herstellung und des Transfers von Kriegsmaterial und der entsprechenden Technologie die internationalen Verpflichtungen und die aussenpolitischen Grundsätze der Schweiz zu wahren; gleichzeitig soll in der Schweiz eine an die Bedürfnisse ihrer Landesverteidigung angepasste industrielle Kapazität aufrechterhalten werden können'. Das bedeutet, dass die oben genannten Aktivitäten bewilligungspflichtig sind, aber nicht wie in der Volksinitiative gefordert verboten werden.

Das Güterkontrollgesetz soll die Ausfuhren von Dual-use-Gütern (zivil und militärisch verwendbar) in Übereinstimmung mit völkerrechtlichen Massnahmen kontrollieren. Das Güterkontrollgesetz ist als Ergänzung des Kriegsmaterialgesetzes gedacht. Ein Artikel des Gesetzes ermächtigt den Bundesrat, die Ausfuhren in Länder, gegen welche die UNO oder die Europäische Union ein internationales Embargo verhängt hat, zu verbieten.

Der Nationalrat und der Ständerat schlossen sich im März bzw. im Oktober dem Vorschlag des Bundesrates an, dem Volk die Ablehnung der Initiative, die insbesondere von der Sozialdemokratischen Partei sowie von zahlreichen Entwicklungsorganisationen und Vereinigungen der Friedensbewegung getragen wurde, zu beantragen. Für die Befürworter der Initiative stellt das von ihnen geforderte Verbot eine grundlegende Voraussetzung für eine kohärente Friedenspolitik dar². Die Gegner wenden ein, dass ein Ausfuhrverbot gravierende wirtschaftliche Folgen, vor allem für die Beschäftigung, zeitigen würde.

In der Diskussion über die Gesetzesrevision ging es im wesentlichen um die Definition des Materials, das zum Geltungsbereich des Gesetzes gehören soll. Im Vorschlag des Bundesrates wurden die Pilatus-Flugzeuge des Typs PC als Kriegsmaterial betrachtet; ferner sollten auch Maschinen und Werkzeuge erfasst werden, die ausschliesslich für die Herstellung oder Wartung von Kriegsmaterial konzipiert sind.

Der Nationalrat und der Ständerat einigten sich auf eine Definition, welche die Pilatus-Flugzeuge aus dem Geltungsbereich des Gesetzes ausklammert. Den meisten Parlamentariern ging es in erster Linie darum, ein bestimmtes Exportunternehmen nicht zu benachteiligen. Es wurde beschlossen, die Ausfuhren dem neuen Bundesgesetz über die Kontrolle zivil und militärisch verwendbarer Güter zu unterstellen. Ende 1996 waren ca. 15 Länder von einem internationalen Embargo betroffen. Die Bewilligungskriterien für Exporte in bestimmte Länder sind aufgrund des Vorschlags des Bundesrats weniger restriktiv als die vorherige bundesrätliche Praxis.

1 Artikel 1 des Bundesgesetzes über das Kriegsmaterial, das vom Parlament am 13. Dezember 1996 verabschiedet wurde.

2 Arguments et commentaires des initiatives populaires pour le désarmement et pour une interdiction des exportations d'armes, Bericht vom Mai 1991, S. 2 (siehe Quellen). 
Tabelle Nr. 16

Schweizerische Kriegsmaterialexporte 1994/1995: Die 15 wichtigsten Abnehmerländer der Schweiz unter den Entwicklungsländern

\begin{tabular}{|c|c|c|c|c|c|}
\hline \multirow[b]{2}{*}{ Land $^{1}$} & \multicolumn{3}{|c|}{1994} & \multicolumn{2}{|c|}{1995} \\
\hline & 1000 Fr. & in $\%$ & Land $^{1}$ & $1000 \mathrm{Fr}$. & in $\%$ \\
\hline Saudiarabien & $54 ' 232$ & 24.5 & Malaysia & $6 ' 558$ & 4.6 \\
\hline Thailand & $27 ’ 570$ & 12.5 & Indien & $5 ’ 318$ & 3.8 \\
\hline Indien & $22 ’ 969$ & 10.4 & Singapur & 4’345 & 3.1 \\
\hline Singapur & 6’671 & 3.0 & Brasilien & $2^{\prime} 129$ & 1.5 \\
\hline Malaysia & 5,420 & 2.5 & Arabische Emirate & 2’090 & 1.5 \\
\hline Bahrain & 3’311 & 1.5 & Argentinien & 1'920 & 1.4 \\
\hline Brasilien & 1'658 & 0.8 & Südkorea & 1'102 & 0.8 \\
\hline Arabische Emirate & 1'637 & 0.7 & Pakistan & 648 & 0.5 \\
\hline Chile & 783 & 0.4 & Marokko & 415 & 0.3 \\
\hline Südkorea & 740 & 0.3 & Tunesien & 297 & 0.2 \\
\hline Marokko & 628 & 0.3 & Bulgarien & 190 & 0.1 \\
\hline Ägypten & 418 & 0.2 & Ägypten & 133 & 0.1 \\
\hline Peru & 370 & 0.2 & Bahrain & 121 & 0.1 \\
\hline Argentinien & 272 & 0.1 & Kroatien & 120 & 0.1 \\
\hline Oman & 240 & 0.1 & Oman & 96 & 0.1 \\
\hline Andere & & & Andere & & \\
\hline Entwicklungsländer & 1’040 & 0.5 & Entwicklungsländer & $1 ’ 256$ & 0.9 \\
\hline $\begin{array}{l}\text { Entwicklungsländer } \\
\text { insgesamt }\end{array}$ & $127 ’ 959$ & 57.9 & $\begin{array}{l}\text { Entwicklungsländer } \\
\text { insgesamt }\end{array}$ & $26^{\prime} 739$ & 18.9 \\
\hline Industrieländer & $93^{\prime} 062$ & 42.1 & Industrieländer & $114^{\prime} 429$ & 81.1 \\
\hline Gesamtexporte & $221^{\prime} 021$ & 100.0 & Gesamtexporte & $141^{\prime} 169$ & 100 \\
\hline
\end{tabular}

1) Endempfängerland.

Quelle: Eidgenössisches Militärdepartement.

Ursprünglich hatte der Ständerat beschlossen, Maschinen und Werkzeuge in den Geltungsbereich des Gesetzes einzubeziehen. Dies betrifft insbesondere Maschinen zur Herstellung von Munition und zum Giessen von Kanonenrohren. Schliesslich aber schwenkte der Ständerat auf die Position des Nationalrats ein, diese Güter aus dem Geltungsbereich des Kriegsmaterialgesetzes herauszunehmen und sie dem Güterkontrollgesetz zu unterstellen. Auch "Ausrüstungsgegenstände, die spezifisch für den militärischen Einsatz oder die Kampfausbildung konzipiert oder dafür abgeändert worden sind"33, hatte das Parlament dem Geltungsbereich des Kriegsmaterialgesetzes entzogen.

Schliesslich sah der bundesrätliche Gesetzesentwurf (Artikel 21) vor, dass der Bundesrat bei der Bewilligung von Auslandsgeschäften insbesondere die Situa-

${ }^{3}$ Artikel 5 des Entwurfs des Bundesrates zum Begriff des Kriegsmaterials. 
tion bezüglich der Menschenrechte sowie die Bestrebungen der Schweiz im Bereich der Entwicklungszusammenarbeit im Bestimmungsland zu berücksichtigen habe. Diese Bestimmung, ein Teil des geltenden Gesetzes, wurde von National- und Ständerat nicht übernommen. Dagegen sieht das neue Gesetz eine Neuerung vor: Transfer und Vermittlung von Technologie sollen abgesehen von möglichen Ausnahmen bewilligungspflichtig sein.

Die Arbeitsgemeinschaft der Hilfswerke war für eine restriktive Haltung im Bereich der Kriegsmaterialausfuhren eingetreten und hatte gefordert, dass die aussenpolitischen Erwägungen vor den Interessen der Rüstungsindustrie Vorrang haben sollen.

Die Gesellschaft zur Förderung der schweizerischen Wirtschaft (wf) ihrerseits plädierte für eine Politik, welche die Schweizer Wirtschaft nicht benachteiligt. Ein in der Parlamentsdebatte häufig geäussertes Argument zugunsten der Lockerung der Bestimmungen über die Kriegsmaterialausfuhr lautete, den exportorientierten Unternehmen dürften keine weiteren Hindernisse in den Weg gestellt werden. Nach Ansicht der wf bedeutete der bundesrätliche Vorschlag eine schwerwiegende Gefahr für den Produktionsstandort Schweiz.

\section{$\square$ Personenminen}

\section{Überprüfungskonferenz der UNO-Waffenkonvention}

Die Überprüfungskonferenz der Vertragsparteien zur Konvention über konventionelle Waffen, die im Jahr 1995 in Wien tagte, führte zur Annahme eines Protokolls über das Verbot von Laser-Blendwaffen (vgl. Jahrbuch Schweiz - Dritte Welt 1996). Die Vertragsparteien konnten sich damals nicht auf eine Verschärfung der international geltenden Einschränkungen von Produktion, Handel und Einsatz von Landminen und anderen unterschiedslos wirkenden Waffen einigen. Die Konferenzarbeiten wurden 1996 in zwei Tagungen vom 15. bis 19. Januar und vom 22. April bis zum 3. Mai in Genf fortgesetzt.

Bei Abschluss der erneuten Überlegungen konnte das Zusatzprotokoll II zur Konvention über Landminen in einigen Punkten leicht verbessert werden. So wird die Anwendbarkeit neben den internationalen auf die nicht internationalen bewaffneten Konflikte erstreckt. Die neuen Regeln, die den Einsatz der Minen einschränken (z.B. durch die Auflage eines Selbstzerstörungs-Mechanismus für Minen) sind jedoch wirkungsarm und recht komplex.

Das Internationale Komitee vom Roten Kreuz (IKRK) sowie zahlreiche Nichtregierungsorganisationen, die zu einem vollständigen Verbot der Personenminen aufgerufen hatten, zeigten sich enttäuscht über die Ergebnisse der Konferenz. Sie wiederholten, dass sich der Einsatz dieser Minen in der Praxis unmöglich regeln lasse und dass ein vollständiges Verbot die einzige Lösung bilde. Zum Zeitpunkt der letzten Tagung der Revisionskonferenz hatten sich rund 30 Länder für ein solches Verbot ausgesprochen.

Im Zusammenhang mit der internationalen Diskussion über die Personenminen liess das IKRK in 21 Ländern Meinungsumfragen durchführen. Die Prozentzahl der Befürworter eines vollständigen Personenminen-Verbots reicht unter den befragten Personen (500 bis 1000 pro Land) von $92 \%$ in Dänemark bis zu $58 \%$ in Japan; in der Schweiz liegt der Anteil bei 88\%. Ferner ergab eine im Auftrag des IKRK von ranghohen Militärs durchgeführte Studie, dass: 
口 es für moderne Berufsarmeen heutzutage praktisch unmöglich ist, die Verwendung von Personenminen mit der Beachtung des Völkerrechts zu vereinbaren;

- die menschlichen und sozialen Kosten von Minen zu der geringen, oft vernachlässigbaren militärischen Wirksamkeit in keinem Verhältnis stehen.

Konferenz von Ottawa über ein vollständiges Verbot von Personenminen

Angesichts der begrenzten Ergebnisse der Überprüfungskonferenz (siehe oben) fanden sich 50 Staaten, internationale Organisationen und NRO auf Einladung Kanadas im Oktober 1996 in Ottawa zusammen, um einen Prozess in Gang zu setzen, der in einem vollständigen Verbot der Personenminen münden sollte. Dank der Konferenz konnten der politische Wille der teilnehmenden Länder gestärkt und rasche Fortschritte ermöglicht werden. Die Konferenz erzielte drei Resultate: i) Schaffung der "Ottawa-Gruppe“, deren 50 Mitglieder sich verpflichten, gemeinsam auf das Verbot und die Beseitigung von Personenminen hinzuarbeiten; ii) Verabschiedung eines Aktionsprogrammes auf globaler und regionaler Ebene; iii) Aufforderung des kanadischen Aussenministers, im Dezember 1997 einen Vertrag über das vollständige Personenminen-Verbot zu unterzeichnen. Ferner enthält die Deklaration von Ottawa das Engagement, zusätzliche Ressourcen für die Entminung, die Betreuung von Minenopfern und die Präventionstätigkeiten bereitzustellen. Die nächste Tagung wird im Juni 1997 in Belgien stattfinden.

Die Schweiz hat sich an der Konferenz aktiv beteiligt und angeboten, eines der nächsten Treffen der "Ottawa-Gruppe" auszurichten.

Haltung der Schweiz in bezug auf die Personenminen

Im September 1995 wurde im Rahmen der nationalen Kampagne gegen Personenminen eine Petition mit 150'000 Unterschriften abgegeben (siehe Jahrbuch Schweiz - Dritte Welt 1996). Im Oktober 1995 kündigte das Eidgenössische Militärdepartement an, dass die Schweiz auf den Gebrauch von Personenminen verzichte; ausgenommen sind solche, die von einem Beobachter ferngezündet werden können. Im Januar 1996 sprach sich die Schweiz anlässlich der Konferenz in Genf für ein vollständiges Verbot aus.

In der Dezembersession beschloss das Parlament, in den Artikeln des Gesetzes über das Verbot von Kernwaffen, biologischen oder chemischen Waffen auch den Verzicht auf Personenminen zu verankern. Schliesslich wurde jedoch eine restriktivere Definition der Personenminen gewählt, als Nationalrat John Dupraz, Genf, anfänglich vorgeschlagen hatte: Panzerabwehrminen, deren Explosion auch von Personen ausgelöst werden kann, bleiben ausserhalb des Geltungsbereichs des Gesetzes.

\section{$\square$ Kohärenz der Politik gegenüber den Entwicklungsländern und Waffenhandel}

Im Rahmen der parlamentarischen Debatte über die Volksinitiative für ein Verbot der Kriegsmaterialausfuhr und über die Revision des Kriegsmaterialgesetzes haben die Hilfswerke die schweizerischen Militärgüterexporte und die Aussenpolitik in einen Zusammenhang gestellt. "Brot für alle" wies im Dossier mit dem Titel "Entwicklung braucht Frieden" nachdrücklich darauf hin, dass Waffenexporte in Länder, welche die Menschenrechte missachten oder in denen eine bürgerkriegsähnliche Situation besteht, den aussenpolitischen Grundsätzen widersprechen. Zudem ist die Rede von den schweizerischen Waffenexporten nach 
Indien und Pakistan, Länder, in denen Spannungen herrschen und die beide zu den Schwerpunktländern der schweizerischen Entwicklungszusammenarbeit gehören.

Der Einsatz von Pilatus-Fluzeugen in Militäraktionen der birmanischen Armee löste bei den Hilfswerken, vor allem bei Swissaid und Caritas, welche dort Flüchtlingsprojekte betreuen, grosse Besorgnis aus. Aufgrund der Lösung des National- und Ständerates können künftig Waffenexporte nach Burma verboten werden, solange das internationale Embargo in Kraft bleibt.

Im gleichen Zusammenhang wies der Nationalrat auf Vorschlag des Bundesrates im März 1996 die Motion Ziegler (SP, GE) zurück. Diese Motion hatte einen Lieferungsstopp für Ersatzteile von PC-7 nach Mexiko gefordert, weil diese Flugzeuge in der Provinz Chiapas eingesetzt würden.

Im übrigen befand das Bundesgericht, dass sich drei leitende Verantwortliche der Firma Von Roll mit der Lieferung von Kriegsmaterial nach Irak, das zur Konstruktion einer "Superkanone" dienen sollte, eines Verstosses gegen das Gesetz über die Ausfuhr von Kriegsmaterial schuldig gemacht hatten. Die Betreffenden wurden zu einer Gefängnisstrafe von einem Monat mit Bewährung verurteilt.

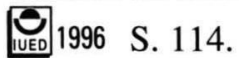

\section{QUELLEN}

Botschaft zur Volksinitiative "für ein Verbot der Kriegsmaterialausfuhr" und zur Revision des Bundesgesetzes über das Kriegsmaterial vom 15. Februar 1995, Bundesblatt 1995 II 1027.

Botschaft betreffend das Bundesgesetz über die Kontrolle zivil und militärisch verwendbarer Güter vom 22. Februar 1995, Bundesblatt 1995 II 1301.

Amtliches Bulletin der Bundesversammlung, Nationalrat und Ständerat, Bern, Sessionen 1996.

Comité romand des initiatives pour la paix et le désarmement, Arguments pour ou contre l'interdiction d'exporter du matériel de guerre, Februar 1996.

Brot für alle, Impulse 2/96, "Entwicklung braucht Frieden“, Bern und Lausanne: PPP, 1996.

Sozialdemokratische Partei der Schweiz und Arbeitsgemeinschaft für Rüstungskontrolle und ein Waffenausfuhrverbot ARW, Arguments et commentaires des initiatives populaires pour le désarmement et pour une interdiction des exportations d'armes, Bern, Mai 1991, 48 S.

Gesellschaft zur Förderung der schweizerischen Wirtschaft (wf), Opinion, Nr. 38, 17. September 1996.

Gesellschaft zur Förderung der schweizerischen Wirtschaft (wf), Documentation, Nr. 41, 8. Oktober 1996.

Internationaler Währungsfonds, Bulletin du FMI, 10. Juni 1996.

Journal de Genève, 29. November 1996.

Neue Zürcher Zeitung, 2. und 16. Februar 1996, 6. und 7. März, 3. und 7. Oktober 1996, 26. November 1996, 5. Dezember 1996.

Tages-Anzeiger, 31. Mai 1996. 


\section{RATIFIZIERUNG DES ÜBEREINKOMMENS DER VEREINTEN NATIONEN ÜBER DIE RECHTE DES KINDES}

Nachdem das Dossier zur Differenzbereinigung dreimal zwischen den beiden Kammern des Parlaments hin- und hergeschoben wurde, war die Schweiz im Dezember 1996 endlich bereit, das Übereinkommen der Vereinten Nationen über die Rechte des Kindes zu ratifizieren.

In seiner Botschaft vom Juni 1994 hatte der Bundesrat die Ratifizierung des Übereinkommens durch die Schweiz empfohlen. Die Frage wurde schliesslich während der Sommer-(Ständerat), Herbst-(Nationalrat) und Wintersession 1996 (Differenzbereinigung zwischen den Kammern) debattiert. Während der Sommersession im Juni hatte der Ständerat die Ratifizierung unter fünf Vorbehalten trotz der Opposition einiger Abgeordneten des rechten Flügels gutgeheissen (35 Stimmen gegen 4). In seiner Botschaft hatte der Bundesrat vier Vorbehalte genannt, die sich gegen die Familienzusammenführung im Falle von Saisonniers, Studenten und Asylbewerbern, den Erwerb der Staatsbürgerschaft durch das Kind, die Trennung von Erwachsenen und Kindern in den Haftanstalten (in einigen Kantonen gibt es noch keine getrennten Gefängnisse) und die Garantien im Zusammenhang mit Strafverfahren richteten (die Garantie auf Vertretung durch einen Anwalt befreit den Angeklagten nicht definitiv von den eventuell entstehenden Kosten; die Trennung zwischen Untersuchungs- und Gerichtsinstanz ist nicht in allen Kantonen gegeben). Der Ständerat fügte seinerseits einen fünften Vorbehalt über die elterliche Gewalt (Art. 5 des Übereinkommens) hinzu, der auf einen Vorschlag von Hans Danieth (CVP) zurückzuführen ist. Dieser Vorbehalt erhielt die Unterstützung der konservativen und nationalistischen Kreise, die "eine Einmischung der Vereinten Nationen in Fragen, die in erster Linie die Verhältnisse innerhalb der Familie betreffen“, befürchteten.

Der Nationalrat stimmte am 1. Oktober 1996 der Ratifizierung einschliesslich der vier vom Bundesrat geäusserten Vorbehalte zu, verwarf aber den fünften, vom Ständerat hinzugefügten Vorbehalt. Nach mehreren Rücküberweisungen zwischen den Kammern akzeptierte der Nationalrat schliesslich am 12. Dezember nach langem Sträuben den fünften Vorbehalt des Ständerates. Die Sprecherin der nationalrätlichen Kommission für Rechtsfragen, Francine Jeanprêtre, betonte, dieses Einlenken habe nur stattgefunden, um die Ratifizierung des Übereinkommens nicht länger hinauszuzögern.

Im März 1996 hatten 86 schweizerische NRO, die sich mit den Rechten des Kindes befassen, einmal mehr auf eine "möglichst baldige Ratifizierung des Übereinkommens" gedrängt. Nachdem die Entscheidung des Parlaments bekanntgeworden war, begrüssten sie die Ratifizierung, bedauerten aber gleichzeitig die angemeldeten Vorbehalte. Die Ratifizierungsurkunden sollen im Frühling 1997 beim Generalsekretär der Vereinten Nationen hinterlegt werden. Die Schweiz ist offiziell das 188. Land, das diesem Übereinkommen beitritt. Damit holt sie einen grossen Rückstand auf, war sie doch zusammen mit den USA, den Vereinigten Arabischen Emiraten, dem Oman, den Cook-Inseln und Somalia eines der fünf letzten Länder, die das Übereinkommen noch nicht ratifiziert hatten. Der Beitritt der Schweiz zum Übereinkommen dürfte im Inland zu Verbesserungen in verschiedenen Bereichen führen: Kinder sollen die Möglichkeit erhalten, in allen sie betreffenden Verfahren wie z.B. einer Scheidung angehört zu werden; die über- 
wiegenden rechtlichen Interessen des Kindes sollen bei Handlungen der öffentlichen Hand berücksichtigt und behinderte und adoptierte Kinder sowie Kinder von Flüchtlingen besser integriert werden. Mit der Ratifizierung des Übereinkommens verfügt die Schweiz über ein universell gültiges rechtliches Instrument, das ihr bei ihrem Einsatz zugunsten der Rechte des Kindes im internationalen Rahmen wertvolle Dienste leisten dürfte.

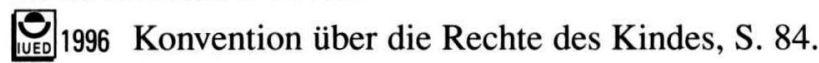

\section{QUELLEN}

Neue Zürcher Zeitung, 6. Juni 1996: "Das Uno-Kinderrechtsabkommen“, 7. Juni 1996: "Der Ständerat ratifiziert Kinderrechtskonvention", 10. September 1996: "Die Schweiz und die Uno-Konvention“, 2. Oktober 1996: "Ja zur Kinderrechtskonvention der UNO".

Tages-Anzeiger, 5. Juni 1996: "Kinder sind die besten Experten".

Journal de Genève, 6. Juni 1996: «Malgré une guérilla juridique, les Etats acceptend la Convention sur les droits de l'enfant», 2. Oktober 1996: «La Suisse pourra ratifier la Convention sur les droits de l'enfant».

La Liberté, 10. September 1996: «Les enfants du monde attendent que la Suisse ratifie leur convention», 14. Dezember 1996: «La Suisse enfin prête à ratifier la Convention des droits de l'enfant».

Nouveau Quotidien, 10. September 1996: «Droit de l'enfant: la Suisse se hâte lentement». 